Cronfa - Swansea University Open Access Repository

This is an author produced version of a paper published in :

Contemporary Nurse

Cronfa URL for this paper:

http://cronfa.swan.ac.uk/Record/cronfa28301

\title{
Paper:
}

Amaniyan, S., Varaei, S., Vaismoradi, M., Haghani, H. \& Sieloff, C. (2016). Effect of local cold and hot pack on the bruising of enoxaparin sodium injection site: a randomized controlled trial. Contemporary Nurse, 52(1), 30-41. http://dx.doi.org/10.1080/10376178.2016.1190289

This article is brought to you by Swansea University. Any person downloading material is agreeing to abide by the terms of the repository licence. Authors are personally responsible for adhering to publisher restrictions or conditions. When uploading content they are required to comply with their publisher agreement and the SHERPA RoMEO database to judge whether or not it is copyright safe to add this version of the paper to this repository. http://www.swansea.ac.uk/iss/researchsupport/cronfa-support/ 


\section{Effect of local cold and hot pack on the bruising of Enoxaparin Sodium Injection Site: A Randomized Controlled Trial}

Sara Amaniyan (MScN, BScN), Shokouh Varaei (PhD, MScN, BScN), Mojtaba Vaismoradi (PhD, MScN, BScN), Hamid Haghani (MScN, BScN) \& Christina Sieloff (PhD, RN)

To cite this article: Sara Amaniyan (MScN, BScN), Shokouh Varaei (PhD, MScN, BScN), Mojtaba Vaismoradi (PhD, MScN, BScN), Hamid Haghani (MScN, BScN) \& Christina Sieloff (PhD, RN) (2016): Effect of local cold and hot pack on the bruising of Enoxaparin Sodium Injection Site: A Randomized Controlled Trial, Contemporary Nurse

To link to this article: http://dx.doi.org/10.1080/10376178.2016.1190289

Accepted author version posted online: 20

May 2016.

Published online: 20 May 2016.

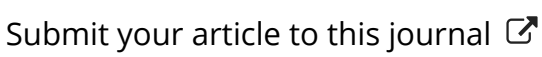

Q View related articles ¿

View Crossmark data \ulcorner 
Publisher: Taylor \& Francis \& Informa UK Limited, trading as Taylor \& Francis Group

Journal: Contemporary Nurse

DOI: $10.1080 / 10376178.2016 .1190289$

\section{Effect of local cold and hot pack on the bruising of Enoxaparin Sodium Injection Site: A Randomized Controlled Trial}

Running head: Bruising prevention and enoxaparin sodium injection

\section{Authors}

1- Sara Amaniyan (MScN, BScN), Faculty of Nursing and Midwifery, Tehran University of Medical Science, Tehran, Iran. E-mail: s-amaniyan@razi.tums.ac.ir

2 - Shokouh Varaei* (PhD, MScN, BScN), Assistant professor, Faculty of Nursing and Midwifery, Tehran University of Medical Science, Tehran, Iran. E-mail: shvaraei@tums.ac.ir

3- Mojtaba Vaismoradi (PhD, MScN, BScN), Researcher, Faculty of Professional Studies, Nord University, Bodø, Norway. Email: mojtaba.vaismoradi@nord.no

4- Hamid Haghani (MScN, BScN), Instructor, School of Health Management and information Sciences, Iran University of Medical Sciences, Tehran, Iran. Email: Haghani@iums.ac.ir

5- Christina Sieloff (PhD, RN), Associate Professor, College of Nursing, Montana State

University, Montana, USA. Email: csieloff@montana.edu

Correspondence: Shokouh Varaei, Faculty of Nursing and Midwifery, Tehran University of Medical Science, Tehran, Iran. E-mail: shvaraei@tums.ac.ir

\section{Declaration of conflicting interests}

No conflict of interest is declared by the authors.

\section{Funding and acknowledgments}

This research was the result of the first author's master degree thesis in nursing science supported financially by the Tehran University of Medical Science. The researchers would like to thank the patients for their participation in this study

\section{Authorship}


All authors of this article have agreed on the final version of this paper and have met all the following criteria: substantial contributions to conception and design, acquisition of data, or analysis and interpretation of data, drafting the article or revising it critically for important intellectual content.

\section{Acknowledgments}

This article is the report of the first author's master degree thesis supported financially by Tehran University of Medical Sciences, Tehran, Iran. The authors wish to thank the patients for their taking part in this research. 


\title{
Effect of local cold and hot pack on the bruising of Enoxaparin Sodium Injection Site: A Randomized Controlled Trial
}

\begin{abstract}
This study aimed to assess the effect of the application of local cold and cold-hot packs upon the size of bruising at the injection site of subcutaneous enoxaparin sodium. One-hundred and eighty patients with coronary diseases were allocated randomly to three groups: (i) local cold gel pack group; (ii) local cold-hot gel pack group; and (iii) a control group who received no apphications of packs. Assessment of the presence of a bruise was conducted at 24,48 and 72 hours after the injections for all patients. The cold-hot pack group had significantlyless (in number) and smaller (in size) injection site bruising than the two other groups at 48 and 72 hours (both $\mathrm{p}<0.001$ ). The local application of cold-hot packs is more effective on reducing bruises following enoxaparin sodium injections when compared with local cold pack application alone. Thus, this process can improve the quality of nursing care for hospitalized patients receiving these injections.
\end{abstract}

Keywords: low-molecular-weight heparin; enoxaparin; injections; bruising; cold application; hot application 


\section{INTRODUCTION}

Medication management encompasses the prescription and administration of drug, and also efforts by nurses for prevention from adverse events that might endanger patient safety. Therefore, management of medicines as an important area of all fields of nursing practice needs especial attention (Hemingway, Baxter, Smith, Burgess-Dawson, Dewhirst, 2011). In this respect, nurses are in a pre-eminent position to enable safe medication management and reduce the probable adverse effects of medication administration (Smeulers, Onderwater, van Zwieten, \& Vermeulen, 2014).

Numerous studies have demonstrated that low-molecular-weight heparins (LMWH), such as enoxaparin sodium, impact thrombus formation and can be used as a part of thromboembolic treatment in cardiovascular patients (Katzung, Masters, \& Trevor, 2012; Dehghani, 2014; Dadaeen, 2015). It has been reported that earlier discharge and no need for partial thromboplastin time (PTT) control have made the application of enoxaparin sodium more economical compared to regular heparin (Dehghani, Najari, \& Dehghani, 2014). However, when enoxaparin is administered subcutaneously, it may also cause adverse effects including pain, local irritation, erythema, hematoma and bruising at the site of injection (Karch, 2010). The incidence levels of local hematomas after LMWH administration is reported between $40 \%$ and $88 \%$, and between 26.6\% and 88.9\%, for bruising (de Campos et al., 2013). These problems are disturbing to patients, can endanger patient safety and result in the patient's avoidance of future injections (Kuzu \& Ucar, 2001). It is important that nurses use strategies to minimize the incidence of above-mentioned adverse outcomes, when administering subcutaneous enoxaparin injections (Chan, 2001), to improve the patients' satisfaction with care and also enhance their trust in health care providers (Chenicek, 2004).

\section{Background}

Various factors influence the occurrence of bruising, hematoma and pain at an injection site after subcutaneous LMWH injections (Kuzu \& Ucar, 2001). Factors that have been investigated by researchers include: 1) injection site (Zeraatkari, Karimi, Shahrzad, \& Changiz, 2008); 2) injection duration (Chan, 2001; Nair, Kaur, \& Sharma, 2008; Zaybak \& Khorshid, 2008); 3) injection volume (Mitchell \& Pauszek, 1987); 4) needle size (Robb \& Kanji, 2002); and 5) the 
application of cold (Kuzu \& Ucar, 2001; Ross \& Soltes, 1995; Varghese, Walia, Sharma, \& Kaur, 2006).

In terms of these adverse effects, the application of cold has been found to have various therapeutic benefits. Cold can help with reducing the severity of pain through decreasing the level of catecholamine, increasing endorphin levels, and delaying the transmission of pain signals to the central nervous system (Ganji, Shirvani, Rezaei-Abhari, \& Danesh, 2013). Cold also constricts the peripheral blood vessels, reduces blood flow to the tissues and decreases the hematoma formation. (Taylor, Lillis, \& LeMone, 2005).

In India, Varghes et al. (2006) examined the impact of moist ice packs on the prevention and reduction of bruising and pain at the 200 heparin injection sites in two intervention and control groups. Their findings supported the use of moist ice packs at 12, 48 and 72 hours following each injection.

Avşar \& Kaşikçi (2013) compared the effect of four injection methods on pain and bruising associated with subcutaneous heparin, 48 and 72 hours after each injection, in 95 hospitalized patients in Turkey. The four methods included: (1) subcutaneous heparin injection without aspiration; (2) subcutaneous heparin injections using airlock, without aspiration; (3) subcutaneous heparin injections with aspiration but without airlock; (4) subcutaneous heparin injections without aspiration, with airlock and two minute cold applications. They showed that the fourth method (adding cold applications to procedures) had a significant influence in the reduction of pain and bruising incidences at both times.

In a randomized controlled trial, Şendir et al. (2015) examined the effect of three methods of injections on 60 patients hospitalized in the orthopedic wards in Turkey: (1) 30-second injection duration, (2) 30-second injection duration with a five minute local dry cold application before and after administration, and (3) a ten second injection duration with no local dry cold application. They observed that the intensity of pain and the occurrence of bruising in the second method was significantly less than the other two methods.

However, other researchers (Kuzu \& Ucar, 2001; Ross \& Soltes, 1995) identified that cold application had no statistically significant impact on the incidence or size of a hematoma at heparin injection sites. Kuzu \& Ucar (2001) studied the effect of cold on the occurrence of hematomas and bruising at the subcutaneous enoxaparin injection sites in three intervention groups (five minute pre-injection application of ice, five minute post-injection application of ice, 
five minute pre and post application of ice) and a control group (no application of ice). Forty eight and 72 hours after the injection, they found no significant differences among the intervention groups or with the control group.

In contrast, the application of heat dilates blood vessels and increases the blood flow to an area, stimulating metabolism. Heat is prescribed for the promotion of muscle relaxation, reduction of edema and inflammation, and the application of medication to wounds (Smith, Duell, \& Martin, 2002). Moreover, midwifery literature has recommended using heat to assist patients to decrease episiotomy ecchymosis and hematoma (Kaur et al., 2013; Mathew, 2013; Pore, 2014).

However, despite the probable positive effects of applying heat to bruises, only one nursing study has been conducted to assess the effects of the application of heat on bruising at a subcutaneous injection site. Balci Akpinar (2013) sought to determine the effect of local dry hot pack applications on the reduction of bruising associated with the subcutaneous injection of heparin in 33 patients. Local dry hot packs were applied on the bruised area for the intervention group 72 hours after the heparin injection. The difference between the bruise size for the intervention and the control groups was statistically significant. Balci Akpinar concluded that the application of heat had a positive effect on the patients' recovery from bruising.

After a thorough literature review, the researchers identified conflicting results related to the effects of the application of cold to bruises of LMHW injections as well as a lack of sufficient studies on the relationship between heat and bruise size associated with enoxaparin therapy. In addition, the researchers did not find studies focusing on the application of cold and hot together on the bruising resulting at a subcutaneous injection. Therefore, the aim of this study was to assess and compare the effects of local applications of cold and cold-hot packs on the bruising at injection sites for subcutaneous enoxaparin sodium. The null hypothesis was that there would be no significant difference observed between the application of local cold and local cold-hot packs on the bruising at injection sites for subcutaneous enoxaparin sodium.

\section{METHODS}

\section{Study design}

This study was designed as a randomized controlled trial using two intervention groups and a third group as a control. The aim of this study was to assess and compare the effects of local 
application of cold and cold-hot packs on the size of bruising at subcutaneous injection sites of enoxaparin sodium. The study was conducted March through May, 2014. Patients with coronary disease, receiving enoxaparin sodium, were selected using a convenience sampling method. The sample size was determined using following formula, where statistical significant was set at $\alpha=$

0.05 and power at 0.80 :

$$
\mathrm{n}=\frac{\left(\mathrm{z} \frac{\mathrm{\alpha}}{2}+\mathrm{z}_{1-\beta_{2}}\right)^{2}\left(\sigma_{1}^{2}+\sigma_{2}^{2}\right)}{\left(\mu_{1}-\mu_{2}\right)^{2}}
$$

To predict an attrition rate of 0.15 , a target sample size of 60 patients was recruited for each group. Equal numbers of females and males were included to prevent gender bias. Patients were allocated randomly to either the intervention or the control group by pulling cards from a package (Polit \& Tatano Beck, 2008). A summary of the study process based on the CONSORT flow diagram (2010) has been presented in Figure 1.

\section{Setting and participants}

The research was conducted on the cardiology wards of a teaching hospital in an urban area of a Middle Eastern country. The inclusion criteria were patients who: 1) were 18 years of age or older; 2) were prescribed enoxaparin sodium injections (60 mg/0.6 ml) twice a day during the treatment process for cardiovascular diseases; 3) did not take any anticoagulant drug except

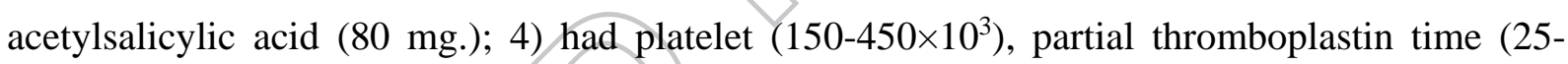
35sec) and international normalized ratio (0.8-1.2) values within the normal limits for beginning enoxaparin sodium injections; 5) had normal renal function (Creatinine: 0.5-1.5 mg/dL, Urea: $15-50 \mathrm{mg} / \mathrm{dL})$; 6) did not have any hematologic disorders or any bruising or injuries at the abdominal wall; 7) received no injections at the abdominal site, other than the enoxaparin sodium, during the research protocol; and 8) did not have any history of skin allergy to the use of local cold or hot packs. Exclusion criteria included the patients who: 1) were pregnant; 2) had bleeding in the injection site; or 3) were not willing to participate in this study.

\section{Measures}

Data collection form had two parts. The first part of the form recorded demographic data such as: age, gender and body mass index. The second part of the form included a table containing: the date, type of group (control, cold pack application, cold/hot packs application), time and location of an injection, and the bruise measurement results at 24, 48 and 72 hours after each injection. 


\section{Injection technique}

Each patient received six doses of enoxaparin sodium injected subcutaneously using a standard protocol by the first researcher. The chosen area was the symmetric points at both the left and right side of the abdomen, five centimeters from the umbilicus. This area was cleansed with an alcohol swab and left to dry before the injection. According to manufacturer's instructions, the skin was pinched, and the full length of the needle (27 gauge syringe) was inserted into the skin fold at a 90-degree angle without ejecting the air bubble prior to the injection or aspiration. The plunger of prefilled syringe was pressed, and the solution was slowly injected, keeping the skin fold pinched throughout this time. Then, the needle was withdrawn and the researcher put a dry swab gently on the spot with no pressure, where the injection was given. The area was not rubbed or massaged. Since some studies have shown a statistically significant difference in reducing the size of bruises by increasing the injection time to 30 seconds (Dadaeen et al., 2015; Balci Akpinar \& Celebioglu, 2008, Babaie Asl, 2008) for all the research groups, the duration of injection was approximately 30 seconds in order to reduce any injection bias. After the injection, a circle with a diameter of five centimeters was drawn around the injection area to avoid using this site for future injections. The researchers assured that the space around the injection site, receiving the application of a pack, had a minimal overlap with the injection site.

Assessment for the presence of a bruise was done after each injection at 24, 48 and 72 hours for all patients in all groups. If a bruise occurred, the longest diameter was measured using a transparent millimeter ruler by an assessor who had was blinded and had no information about the patient's group, and the researcher recorded the data on the collection form. This use of the assessor and the researcher was done to reduce measurement bias. The bruise size was categorized using the same classification as Varghes et al.'s (2006) study: a large bruise was larger than five millimeters, and a small bruise was between two to five millimeters. The researcher determined that no bruise existed if the bruise was less than two millimeters. The overall agreement between the researcher and the assessor on the diameters of the bruises, was 0.86 , using kappa test agreement. 


\section{Application of the gel packs}

\section{Equipment}

The equipment used in this study consisted of: 1) reusable cold/hot packs (PMB ${ }^{\circledR}$, Tehran, Iran) (size: $15 \times 11 \mathrm{~cm}$ ); 2) a thermometer to determine the temperature of the packs; 3 ) disposable towel bags to cover reusable cold/hot packs; 4) a watch with a second hand to

measure the duration of an enoxaparin sodium injection and the duration of the applications of cold and cold/hot packs; 5) a waterproof pen to mark the injection site; and 6) a millimeter transparent ruler for measuring the longest dimension of a bruise.

\section{Procedure}

The procedure for each group adhered to the following processes:

(i) local cold gel pack group. A reusable cold gel pack, covered with a fabric towel bag, was applied to the injection site for twenty minutes (Taylor et al., 2005; Smith et al., 2002) immediately after each injection. The patient's skin at the injection site was checked every five minutes to prevent it from skin damage during the application of the cold (Taylor et al., 2005; Smith et al., 2002). The temperature range of these packs was $0-2^{\circ} \mathrm{C}$ based on the instructions recommended by available nursing textbooks (Taylor et al., 2005);

(ii) local cold-hot gel packs group. In addition to the application of a cold gel pack on the injection site for 20 minutes after every injection, a reusable hot gel pack was also placed on the same injection site, 12 hours after the time of the injection. A hot pack was applied on the bruised area to eliminate the risk of bleeding 12 hours after the administration of the cold pack that corresponded with the duration of action of enoxaparin (Karch, 2010). A fabric towel bag, with a hot pack, was applied for 20 minutes (Taylor et al., 2005; Smith et al., 2002). The temperature range of these packs was $40-42^{\circ} \mathrm{C}$ based on the instructions recommended by available nursing textbooks (Taylor et al., 2005). Throughout the entire process, the patient's skin, at the injection site, was checked every five minutes to prevent it from skin damage (Taylor et al., 2005; Smith et al., 2002).

(iii) control group. Patients in this group did not receive any special intervention beyond that of the routine injection of subcutaneous enoxaparin sodium. 


\section{Ethical considerations}

This study is the report of the first author's master degree thesis. The investigation conforms with the principles outlined in the Declaration of Helsinki. Ethical permissions for the study was granted by the Ethics Committee of the university where the researchers were employed (ethical code: 92/130/2597). The purpose and procedures of the research were explained to the patients and written informed consent was obtained from them prior to their participation. The patients were informed of their rights to terminate their participation at any time throughout the study without facing any sort of penalty or impact on their routine care during hospitalization. They were assured that their names would not be disclosed, and they could request a report of the study findings. Also, this clinical trial has been registered in the required website of Registry of Clinical Trial with the Registration code of IRCT2014040917197N1.

\section{Data analysis}

The data were analyzed using the SPSS software, version 16.0 for Windows (SPSS Inc., Chicago, IL, USA). Descriptive statistics were used to characterize the study population by applying frequencies and percentages for qualitative variables, and means and standard errors for quantitative variables. Within all stages of analysis, the significance level was set at $\alpha=0.05$.

The normality of the distribution of the dependent variable (size of bruising) was examined using the Kolmogorov-Smirnoy test $(p<0.05)$. To investigate whether the interventions affected the dependent variable at different times, repeated measures of analyses of variance (RANOVA) were used.

\section{RESULTS}

\section{Demographical characteristics of patients}

In this research, there were 30 male patients (50\%) and 30 female patients (50\%) in each group in an attempt to control for gender bias. The mean \pm SD age of the patients were: 1) cold pack group: $59.58 \pm 1.68,2$ ) cold-hot pack group: $54.72 \pm 1.61$, and 3) control group: $59.53 \pm$ 1.93. In addition, the average BMI for the cold pack group, cold-hot pack group and control group were $25.19 \pm 0.43,24.73 \pm 0.45$, and $23.43 \pm 0.46$, respectively. No significant difference was found between the size of the bruise and demographic information in the groups (Table 1). 


\section{Effect of interventions on the size of bruising}

The results of ANOVA, using a post hoc Tukey's Honestly Significant Difference (HSD), for the size of the bruising demonstrated significant differences between the groups. The average size of bruising in the cold pack group and the cold-hot pack group were significantly less (p 0.001) than the size of the bruising in the control group. Additionally, no large bruise was observed in the patients of the cold-hot pack group at any of three time (Table 2).

The percent of 'no bruise' in the cold pack group at 48 and 72 hours (26.7\% and 46.7\%) was much lower than this amount in cold-hot pack group (55.0\% and 78.3\%) after injections ( $\mathrm{p}<$ 0.001). However, at 24 hours after the injection, the average bruising size between the cold pack group $(70 \%, 1.57 \pm 0.09 \mathrm{~mm})$ and cold-hot pack group $(88.3 \%, 1.22 \pm 0.07 \mathrm{~mm})$ were similar and there was no statistically significant difference between them $(p=0.19)$. But the cold-hot pack group had significantly less (in number) and smaller (in size) injection site bruising than the two other groups at 48 and 72 hours (both $\mathrm{p}<0.001$ ).

\section{Effect of time (24, 48 \& 72 hours after the injections) on the size of bruising}

The results of RANOVA, and post hoc tests using the Bonferroni correction (SPSS Inc., Chicago, IL, USA) revealed that the average size of bruising for all three groups, on the first day of study, was significantly less than the amount of bruising on the second and third days $(\mathrm{p}<0.001, \mathrm{p}<0.001)$.

In the cold pack and cold-hot pack groups, the average size of bruising on third day was significantly less than the bruising on the second day $(\mathrm{p}<0.001)$. In contrast, the average bruising size on day 3 , in the control group was significantly increased as compared to $2^{\text {nd }}$ day $(\mathrm{p}<0.001)$ (Table 2). Figure 2 displays the effect of time and treatment on bruising size in 3 groups.

As observed in all groups 24 to 48 hours after the injection, the average size of bruising increased and then the bruising size for both intervention groups was reduced until 72 hours after the injection. However, during this time, for the control group, the average size of bruising was gradually increasing. It seems that applying local cold and hot pack in the intervention groups reduced the growth of bruising. Whereas, in the control group, after a sudden increase of bruising size, only the rate of expansion of the bruise decreased. 


\section{DISCUSSION}

The findings of this study rejected the null hypothesis (no significant difference was observed between the local application of cold and cold-hot packs on the resulting bruising at injection sites for subcutaneous enoxaparin sodium.

Our results showed that both the interventions were effective but the local application of coldhot packs, rather than the application of a cold pack alone, was more effective in the reduction of the bruises of subcutaneous enoxaparin injections. However, there were no significant differences between the bruise sizes of the two interventions 24 hours after the injections.

The results of present study supported Akpinar's (2013) findings that the size of bruising, as a result of receiving heparin injections, for a group of patients receiving a hot pack was smaller in comparison to the control group $(\mathrm{p}<0.05)$. Her research demonstrated that the local hot, used in the intervention group, increased local blood circulation and removed blood cells accumulated under the skin as a possible physiological mechanism in minimizing bruise size.

Additionally, Varghes et al. (2006) demonstrated the significant effect of cold in reducing the size of injection site bruising. They reported that there was a decrease in size for the large and small bruises in the intervention group as compared with the control group at 12, 48 and 72 hours after the injections. The subjects, in the intervention group, also experienced less bruising than the subjects in the control group $(\mathrm{p}<0.05)$. Our study findings also supported Avsar \& Kasikçi’s (2013) findings in terms of the effectiveness of cold packs in reducing the size of bruising. They showed a significant difference between the heparin injection methods by applying cold packs versus injection methods with no cold pack application. However, the studies by Kuzu \& Ucar (2001) and Ross \& Soltes (1995) showed no statistically significant difference between incidence of bruising and applying of local cold packs on the injection site. Perhaps, the most important reasons for these controversial results can be the differences in duration of cold pack applications, temperature of cold packs and/or research designs.

In the current study, for both intervention groups, the peak of bruising was at 48 hours after injections, and the beginning of the disappearing process was within 24 hours after this peak. This finding was consistent with the finding of previous studies (Chan, 2001; Balci Akpinar \& Celebioglu, 2008; Varghes et al., 2006, de Campos, 2013; Dadaeen, 2015). However, contrary to the findings of those studies, the current study's control group also demonstrated increasing bruise size 72 hours after the injections. 
It was surprising that, in this study, regardless of the type of the patient groups, differences in the mean sizes of the bruises, when compared with those reported in previous studies, were found much smaller. For example $3.21 \pm 3.78 \mathrm{~cm}^{2}$ in the intervention group and $5.22 \pm 4.54 \mathrm{~cm}^{2}$ in the control group for findings of the Balci Akpinar's study, $109.20 \mathrm{~mm}^{2}$ at 48 hours and $110.12 \mathrm{~mm}^{2}$ at 72 hours in the control group, and 18.76 and $21.72 \mathrm{~mm}^{2}$, in the interventions group for findings of Zaybak and Khorshid's study, $5.16 \mathrm{~mm}$ in the intervention group and 5.44 $\mathrm{mm}$ in the control group for findings of Klingman's study (2000). This may be due to the combination of factors influencing the size of bruises such as a slow administration of enoxaparin injections without aspiration, the use of an air bubble and a finer gauge needle. In addition, this study investigated the size of bruises not the size of hematomas. As a consequence, because of the small sizes of bruises in this study, despite significant differences between intervention groups, the findings were very close together.

In nursing literature, there is only one study that has evaluated the effect of the local hot pack application on the bruising of the heparin injection site, 72 hours after each injection. As a result,, a strength of the current research is of the findings related to the application of hot packs at the first 12 hours after each injection of enoxaparin sodium without any complication for coronary patients, while also comparing the: (1) efficacy of the local applications of cold and hot packs together, to (2) the application of cold packs alone, to (3) a control group for subcutaneous injections of enoxaparin.

\section{Limitations and suggestions for future studies}

A limitation of this study was a lack of a similar standardized method for the combined application of local cold and hot packs on subcutaneous LMHW injection sites. As a result, the framework of method had to be designed by the researchers according to available nursing literature.

Another limitation was a lack of detailed information to compare the bruising size at enoxaparin injection sites in the current study with previous researches. One reason for this lack of information was due to reported differences in the measurement scales used. In some previous studies, the bruises were measured in millimeter ${ }^{2}$ or centimeter $^{2}$ because of the extent of bruising area. But in the current study, due to the appearance of small bruises, only the maximum diameter of the bruises in millimeters was measured. 
The current study was conducted to assess the bruising size after the injection of enoxaparin sodium (60 mg/0.6 ml) for patients with coronary diseases in cardiology wards. Thus, it is recommended that further studies be conducted using different settings with different dosages, using the same methods in order to increase the generalizability of the findings to different groups of patients.

\section{CONCLUSION}

The results of the current research indicated that the application of local cold and hot packs lead to a decrease in the size and frequency of bruising following injections of 60 milligrams of enoxaparin sodium. In addition, the combined application of local cold and hot enhanced the effectiveness of the intervention.

Given the positive impact of this intervention, consideration may be given to this approach as a strategy to preserve patient safety through elimination or reduction of post-injection bruising related to injections of enoxaparin sodium. Implementation of this strategy is inexpensive and easy to use, can preserve patient safety and also improve the quality of nursing care from the physical aspects for hospitalized patients in cardiology wards and so it has important clinical implications for nursing practice.

\section{REFERENCES}

Avsar, G., \& Kasikçi, M. (2013) Assessment of four different methods in subcutaneous heparin applications with regard to causing bruise and pain. International Journal of Nursing Practice, 19, 402-408.

Babaie Asl, F. (2008) Effect of injection duration on bruise size associated with subcutaneous heparin. Journal of Babol University of Medical Sciences, 10, 49-55.

Balci Akpinar, R. (2013) The effect of local dry heat pack application on recovering the bruising associated with the subcutaneous injection of heparin. Journal of Clinical Nursing; 22, 25312535.

Balci Akpinar, R., \& Celebioglu, A. (2008) Effect of injection duration on bruising associated with subcutaneous heparin: A quasi-experimental within-subject design. International Journal of Nursing Studies, 45, 812-17.

Barrett, K., Brooks, H., Boitano, S., \& Barman, S. (2010) Ganong's Review of Medical Physiology. 23rd. McGraw-Hill, p. 535. 
Chan, H. (2001) Effect of injection during on site-pain intensity and bruising associated with subcutaneous heparin. Journal of Advanced Nursing, 35, 882-892.

Chenicek, T. E. (2004) Effects of injection duration on site-pain intensity and bruising associated with subcutaneous administration of lovenox (enoxaparin). Master's Thesis, The Florida State University School of Nursing, Retrieved from http:// diginole.lib.fsu.edu/cgi/viewcontent.

Dadaeen, A., Bahreini, M., Bazi, P., Ostovar, A., Raeisi, A., Dobaradaran, S. (2015) The Effect of Duration of Subcutaneous Injection on the Extent of Bruising and Pain Intensity at Injection Sites among Patients Receiving Enoxaparin Sodium: A Randomized Self-Controlled Clinical Trial. Int Cardiovasc Res J, 9, 77-82.

de Campos, JDF., da Silva, JB., Beck, ARM., Secoli, SR., de Melo Lima, MH. (2013) Subcutaneous administration technique of low-molecular-weight heparins: An integrative review. Clinical Nursing Studies, 1, 36-44.

Dehghani, K.H., Najari, Z., Dehghani, H. (2014) Effect of subcutaneous Enoxaparin injection duration on bruising size in acute coronary syndrome patients. Iran J Nurs Midwifery Res, 19, $564-568$.

Ganji, Z.H., Shirvani, MA., Rezaei-Abhari, F., Danesh, M. (2013) The effect of intermittent local heat and cold on labor pain and child birth outcome. Iran J Nurs Midwifery Res, 18, 298303.

Hemingway, S., Baxter, H., Smith, G., Burgess-Dawson, R., \& Dewhirst K. (2011) Collaboratively planning for medicines administration competency: a survey evaluation. Journal of Nursing Management, 19, 366-376.

Katzung, B. G., Masters, S. B., \& Trevor, A. J. (2012) Basic \& Clinical Pharmacology. 12th. McGraw-Hill, p. 605.

Karch, A. M. Lippincott's Nursing Drug Guide. China: Lippincott Williams \& Wilkins pub, 2010, p.438.

Kaur, N., Kaur Rana, A., Suri,V. (2O13) Effect of dry heat versus moist heat on Episiotomy pain and wound healing. Nursing and Midwifery Research Journal, 9, 21-33.

Klingman, L. (2000) Effects of changing needles prior to administering heparin subcutaneously. Heart \& Lung, 29, 70-75. 
Kuzu, N., Ucar, H. (2001) The effect of cold on the occurrence of bruising, hematoma and pain at the injection site in subcutaneous low molecular weight heparin. International Journal of Nursing Studies, 38, 51-59.

Mathew, J. (2013) A Study to evaluate the effectiveness of cold before warm versus only warm application in reduction of perineal discomfort among postpartum mothers in selected maternity units, Bangalore. Master’s Thesis, University of Health Science Bangalore, Karnataka.

Mitchell, G.S., Pauszek, M. (1987) Effect of injection volume on local hematoma formation during Low-Dose heparin therapy. Critical Care Medicine, 44, 87-88.

Nair, P., Kaur, S., Sharma, Y.P. (2008) Effect of time taken in injecting subcutaneous heparin injection with reference to site pain and bruising among patients receiving heparin therapy. Nursing and Midwifery Research Journal, 4, 7-15.

Polit, D.F., Tatano Beck, C. (2008) Essentials of Nursing Research. 7th. Philadelphia: Lippincott Williams \& Wilkins, pp. 226.

Pore, Y. (2014) Effectiveness of moist heat and dry heat application on healing of episiotomy wound. Asian Journal of Multidisciplinary Studies, 2, 225-236.

Robb, D.M., Kanji, Z. (2002) Comparison of two needle sizes for subcutaneous administration of Enoxaparin: effects on size of hematomas and pain on injection. The Journal of Human Pharmacology and Drug Therapy, 22, 1105-1109.

Ross, S., Soltes, D. (1995) Heparin and haematoma: does ice make a difference. Journal of Advance Nursing, 21, 434-439.

Şendir, M., Büyükyilmaz, F., Çelik, Z., Taşköprü, İ. (2015) Comparison of 3 methods to prevent pain and bruising after subcutaneous heparin administration. Clinical Nurse Specialist, 29, 174180.

Smeulers, M., Onderwater, A.T., van Zwieten, M.C.B., \& Vermeulen, H. (2014) Nurses' experiences and perspectives on medication safety practices: an explorative qualitative study. Journal of Nursing Management, 22, 276-285.

Smith, S.F., Duell, D.J., Martin, B.C. (2002) Photo Guide of Nursing Skills. New Jersey: Pearson Education, Inc., Upper Saddle River.

Taylor, C., Lillis, C., LeMone, P. (2005) Taylor's clinical nursing skills: a nursing process approach. 5th. Philadelphia: Lippincott Williams \& Wilkins, pp. 299-314. 
Varghese, C., Walia, I., Sharma, Y.P., Kaur, S. (2006) Prevention and reduction of pain, bruise and hematoma by Moist Ice Pack application on the site of subcutaneous heparin injection. Nursing and Midwifery Research Journal, 12, 139-148.

Zaybak, A., Khorshid, L. (2008) A study on the effect of the duration of subcutaneous heparin injection on bruising and pain. Journal of Clinical Nursing, 17, 378-385.

Zeraatkari, K., Karimi, M., Shahrzad, MK, Changiz, T. (2005) Comparison of heparin subcutaneous injection in thigh, arm and abdomen. Canadian Journal of Anesthesia, 42, 52-60. 
Table 1. Demographical characteristics of the patients in groups

\begin{tabular}{clll}
\hline Characteristics & Cold (n= 60) & Cold-hot (n= 60) & Control (n = 60) \\
\hline Gender, n (\%) & & & \\
Male & $30(50.0 \%)$ & $30(50.0 \%)$ & $30(50.0 \%)$ \\
Female & $30(50.0 \%)$ & $30(50.0 \%)$ & $30(50.0 \%)$ \\
& & & P-Value \\
\hline Age, n (\%) & & & $3(5 \%)$ \\
$<=40$ & $4(6.7 \%)$ & $35(58.3 \%)$ & $32(53.3 \%)$ \\
$41-60$ & $31(51.6 \%)$ & $16(26.7 \%)$ & $21(35 \%)$ \\
$61-80$ & $22(36.7 \%)$ & $3(5.0 \%)$ & $5(6.7 \%)$ \\
$>80$ & $3(5.0 \%)$ & $54.72 \pm 1.61$ & $3(5.0 \%)$ \\
Mean \pm SD & $59.58 \pm 1.68$ & $2(3.3 \%)$ & $41(68.3 \%)$ \\
BMI, n (\%) & $3(5.0 \%)$ & $34(56.7 \%)$ & $12(20.0 \%)$ \\
$<18.5$ & $33(55 \%)$ & $18(30.0 \%)$ & $4(6.7 \%)$ \\
$18.5-24.9$ & $20(33.3 \%)$ & $6(10.0 \%)$ & $23.43 \pm 0.46$ \\
$25-29.9$ & $4(6.7 \%)$ & $24.73 \pm 0.45$ & \\
$>=30$ & $25.19 \pm 0.43$ & &
\end{tabular}

$*$ BMI $=$ Body Mass Index

${ }^{*}$ p-values were obtained based on Chi square test and one way ANOVA

Table 2. Comparison of mean bruise size (mm) between groups 


\begin{tabular}{|c|c|c|c|c|}
\hline $\begin{array}{l}\text { Treatment } \\
\text { Bruising }\end{array}$ & Cold $(n=60)$ & Cold-hot $(n=60)$ & Control $(n=60)$ & $\begin{array}{l}\text { Test Statistics } \\
\text { p-value }\end{array}$ \\
\hline \multicolumn{5}{|l|}{$24 \mathrm{~h}, \mathrm{n}(\%)$} \\
\hline No Bruise & $42(70.0 \%)$ & $53(88.3 \%)$ & $31(51.7 \%)$ & $F(2,177)=25.04$ \\
\hline Small Bruise & $18(30.0 \%)$ & $7(11.7 \%)$ & $27(45.0 \%)$ & $\mathrm{P}<0.001$ \\
\hline Large Bruise & $0(0.0 \%)$ & $0(0.0 \%)$ & $2(3.3 \%)$ & \\
\hline Mean \pm SD & $1.57 \pm 0.09$ & $1.22 \pm 0.07$ & $2.40 \pm 0.18$ & \\
\hline \multicolumn{5}{|l|}{$48 h, n(\%)$} \\
\hline No Bruise & $16(26.7 \%)$ & $33(55.0 \%)$ & $1(1.7 \%)$ & $F(2,177)$ \\
\hline Small Bruise & $43(71.7 \%)$ & $27(45.0 \%)$ & $39(65.0 \%)$ & $\mathrm{P}<0 \times 1$ \\
\hline Large Bruise & $1(1.6 \%)$ & $0(0.0 \%)$ & $20(33.3 \%)$ & \\
\hline Mean \pm SD & $2.75 \pm 0.13$ & $1.97 \pm 0.11$ & $4.35 \pm 0.24$ & \\
\hline \multicolumn{5}{|l|}{ 72h, n (\%) } \\
\hline No Bruise & $28(46.7 \%)$ & 47 (78.3\%) & $1(1.7 \%)$ & \\
\hline Small Bruise & $32(53.3 \%)$ & $13(21.7 \%)$ & 38 (63.3\%) & \\
\hline Large Bruise & $0(0.0 \%)$ & $0(0.0 \%)$ & $21(35.0 \%)$ & \\
\hline Mean \pm SD & $2.19 \pm 0.12$ & $1.37 \pm 0.09$ & $4.45 \pm 0.23$ & \\
\hline Test Statistics & $\begin{array}{l}F(1.40,82.39)=193.51 \\
P<0.001\end{array}$ & $\begin{array}{l}F(1.42,83.85)=87.86 \\
P<0,001\end{array}$ & $\mathrm{~F}(1.76,103.97)=1$ & \\
\hline
\end{tabular}

*p-values were obtained using ANOVA and RANOVA tests 


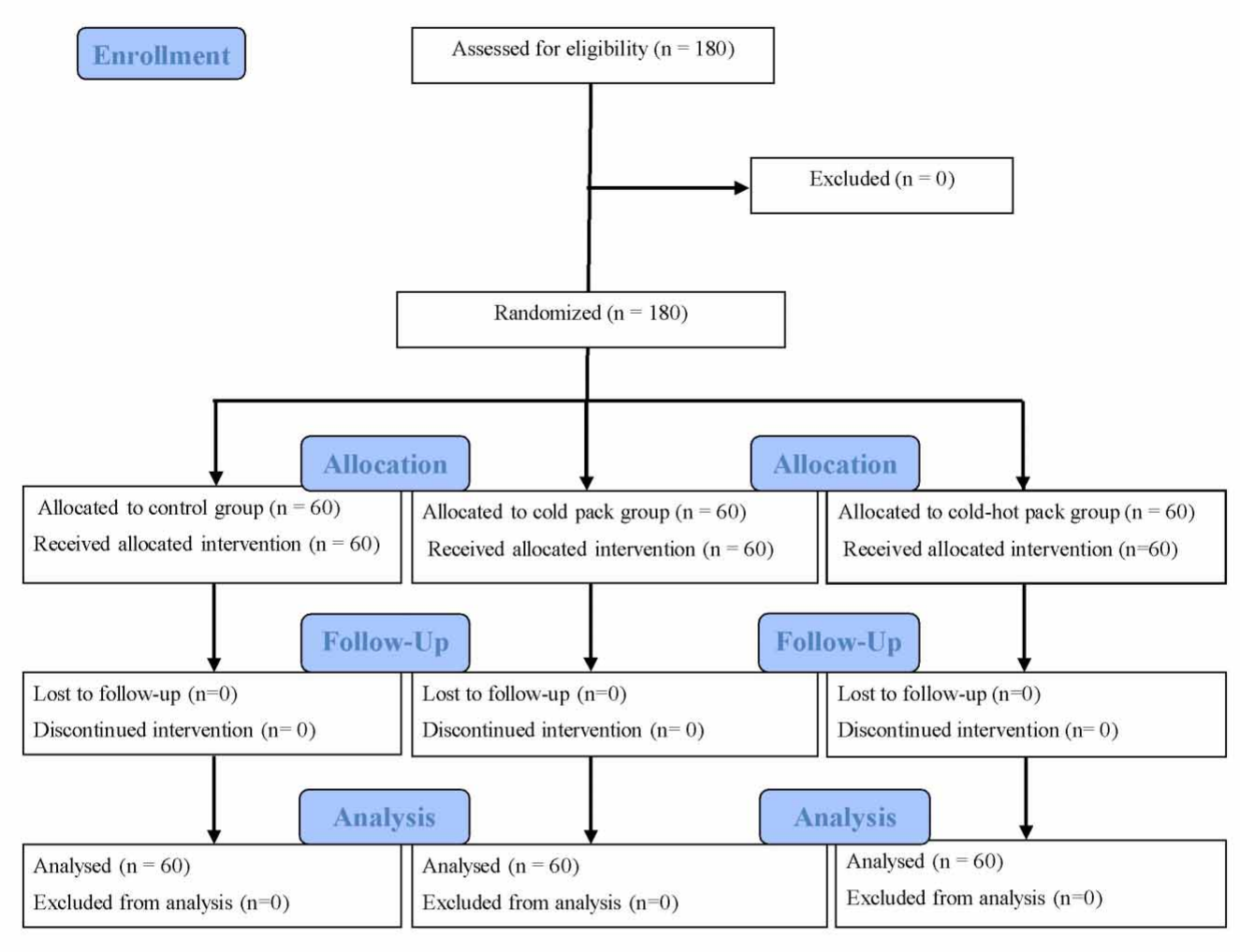

Fig 1. The process of study according to the Consort flow diagram (2010) 


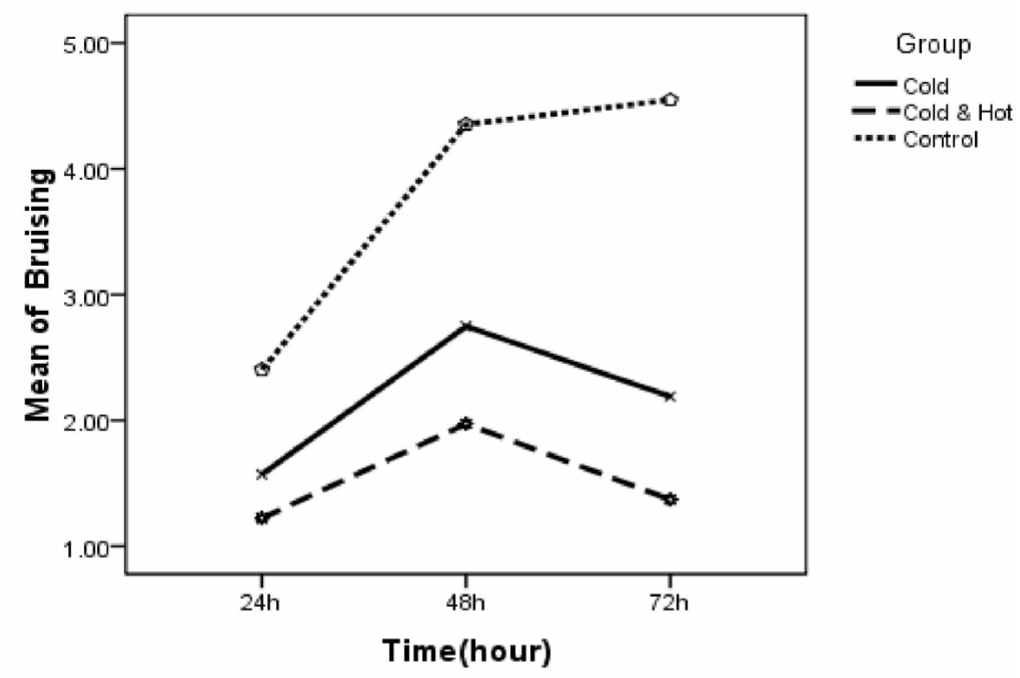

Fig 2. The mean bruise size ( $\mathrm{mm})$ at different times after the injections in each group 